\title{
Selective bond breakage within the HOD molecule using optimized femtosecond ultraviolet laser pulses
}

Tiwari, Ashwani Kumar; Møller, Klaus Braagaard; Henriksen, Niels Engholm

Published in:

Physical Review A

Link to article, DOI:

10.1103/PhysRevA.78.065402

Publication date:

2008

Document Version

Publisher's PDF, also known as Version of record

Link back to DTU Orbit

Citation (APA):

Tiwari, A. K., Møller, K. B., \& Henriksen, N. E. (2008). Selective bond breakage within the HOD molecule using optimized femtosecond ultraviolet laser pulses. Physical Review A, 78(6), 065402.

https://doi.org/10.1103/PhysRevA.78.065402

\section{General rights}

Copyright and moral rights for the publications made accessible in the public portal are retained by the authors and/or other copyright owners and it is a condition of accessing publications that users recognise and abide by the legal requirements associated with these rights.

- Users may download and print one copy of any publication from the public portal for the purpose of private study or research.

- You may not further distribute the material or use it for any profit-making activity or commercial gain

- You may freely distribute the URL identifying the publication in the public portal 


\title{
Selective bond breakage within the HOD molecule using optimized femtosecond ultraviolet laser pulses
}

\author{
Ashwani Kumar Tiwari, Klaus B. Møller, and Niels E. Henriksen \\ CMM, Department of Chemistry, Building 207, Technical University of Denmark, DK-2800 Kgs. Lyngby, Denmark
}

(Received 23 June 2008; published 16 December 2008)

\begin{abstract}
With the HOD molecule initially in its vibrational ground state, we theoretically analyze the laser-induced control of the $\mathrm{OD} / \mathrm{OH}$ branching ratio $\mathrm{D}+\mathrm{OH} \leftarrow \mathrm{HOD} \rightarrow \mathrm{H}+\mathrm{OD}$ in the first absorption band. In the weak-field limit, any form of UV-pulse shaping control leads to a branching ratio larger than $\sim 2$. We obtain in the strong-field limit (peak intensities $\sim 10 \mathrm{TW} / \mathrm{cm}^{2}$ ) a branching ratio significantly less than 2 . The optimized pulses operate by a pump-dump-pump mechanism, where the dumping to the electronic ground state creates nonstationary vibrational states in HOD.
\end{abstract}

DOI: 10.1103/PhysRevA.78.065402

PACS number(s): 32.80.Qk, 82.20.-w, 82.53.-k, 82.50.Nd

The idea that optimized laser fields can guide the dynamics of an atom or molecule from a given initial state into a desired final state has attracted much attention in recent years [1-3]. Processes of particular interest lead via nontrivial laser fields to final states that are not accessible by conventional (photo)chemical means. In current experiments [4-8] laser fields with complex pulse shapes are created via feedback from an experimental signal. The typical situation in these experiments is that the photoinduced dynamics of a molecule, initially in its vibrational ground state, is controlled via optimization of the laser pulse.

An interesting objective is to use the concept of optimized laser fields to obtain selective bond breakage in polyatomic molecules. So far this problem has only been theoretically investigated for model systems and with a few notable exceptions (see, e.g., Ref. [9]) the understanding of the mechanism underlying experimentally obtained complex pulse shapes is so far quite limited.

In this paper we consider the HOD molecule. The weakfield UV (ultraviolet) photodissociation dynamics of HOD in its first absorption band is very well understood. This, and the obvious simplicity of the molecule, has made HOD the "hydrogen atom" in the field of laser-controlled selective bond breakage [10-19]. Thus, it offers a unique opportunity to study optimized laser fields and the associated mechanisms. Previous investigations have shown that, starting with HOD in its vibrational ground state, it is not straightforward to obtain preferred cleavage of the O-D bond by UV excitation [19]. We show here that through careful optimization of the time-frequency behavior of intense UV pulses, it is indeed possible to obtain preferred cleavage of the O-D bond. We also provide an interpretation of the mechanism behind the results.

The two-body dissociation of HOD in the first absorption band is a simple example of a photoinduced reaction with chemically distinct products

$$
\mathrm{HOD} \rightarrow\left\{\begin{array}{l}
\mathrm{H}+\mathrm{OD}, \\
\mathrm{D}+\mathrm{OH}
\end{array}\right.
$$

This reaction is a prototype system for direct dissociation on a single isolated potential energy surface, see Fig. 1.
High-quality potential energy surfaces for the ground and the first excited electronic states including a transition-dipole function between these states are available. The dynamics can be described within a well established two-degree of freedom model where bending and rotation are neglected. With HOD in its vibrational ground state, it has been found in theoretical [20-22] as well as experimental studies [23] using ultraviolet $\mathrm{cw}$ lasers in the weak-field limit, that bond breaking of H-OD is preferred over the breaking of HO-D. The branching ratio depends quite strongly on the wave length within the first absorption band (with a maximum at $\lambda \sim 166 \mathrm{~nm}$ ) but it is always found that the branching ratio $\mathrm{OD} / \mathrm{OH}$ is larger than 1.9.

The majority of the previous works on control have focused on branching ratio control following bond-selective

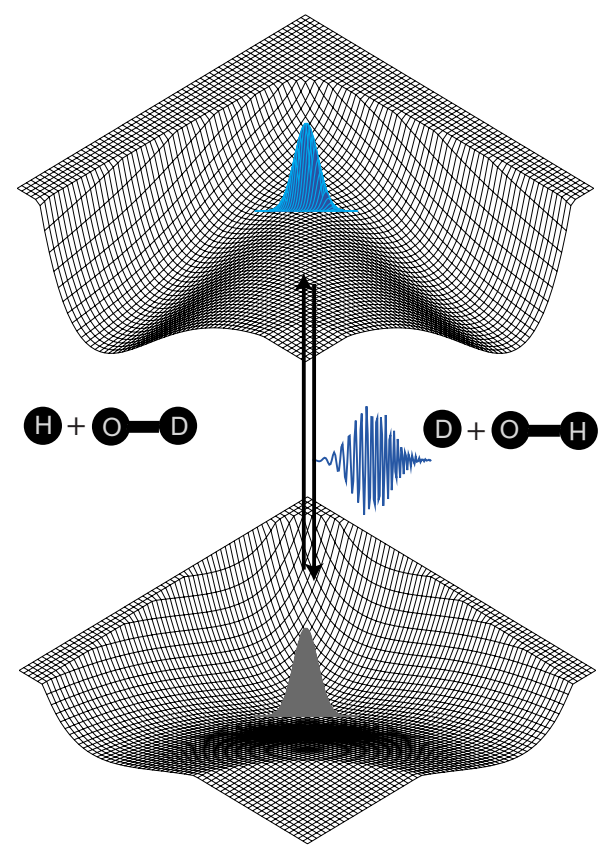

FIG. 1. (Color online) The ground and first excited state potential energy surfaces for water. The two potentials asymptotically approach the same value, but we have here separated the surfaces for graphical purposes. The Franck-Condon wave packet is shown right after an instantaneous excitation. 
vibrational preexcitation of HOD. That is, schemes involving IR (infrared) plus UV excitation. Spurred by this interest and by current experiments with optimally tailored laser pulses [4-8] we show that it is possible to obtain a branching ratio less than 1.9, even without vibrational preexcitation of HOD.

In the weak-field limit, described by first-order perturbation theory for the interaction with the laser field, amplitude is transferred exclusively from the ground state to the excited state surface. The branching ratio has been analyzed for Gaussian pulses as a function of the full width at half maximum (FWHM) [19]. It was found that a pulse with a center frequency corresponding to the smallest branching ratio and temporal width of about $120 \mathrm{fs}$ gives $\mathrm{OD} / \mathrm{OH}=1.90$, in agreement with previous results in the $\mathrm{cw}$ limit [21]. Shorter pulses give a higher branching ratio. For example, a $3 \mathrm{fs}$ Gaussian pulse gives a branching ratio close to 3 which is close to the result obtained for a pulse infinitely short in time, a $\delta$ pulse.

For dissociation out of the vibrational ground state of HOD, the mechanism behind this preferred cleavage of the $\mathrm{O}-\mathrm{H}$ bond has been analyzed. Briefly, due to the smaller reduced mass associated with the $\mathrm{O}-\mathrm{H}$ motion, the probability density in coordinate space moves faster into the $\mathrm{H}$ $+\mathrm{OD}$ channel than into the $\mathrm{D}+\mathrm{OH}$ channel-the final branching ratio is, however, strongly modified by the initial momentum distribution [22].

While pulse shaping in the weak-field limit can lead to interesting phenomena such as focusing of continuum wave packets on repulsive potential energy surfaces [24], it can be shown, in accordance with the results reported for the Gaussian pulses, that the pulse shape in general has a rather "trivial" effect on the branching ratio [25-27]. In the final product distributions obtained with shaped laser pulses, a number of energy states are simultaneously excited, but in such a way that the same distribution could have been obtained via a set of consecutive (incoherent) processes induced by cw lasers with the same frequency distribution as in the shaped pulse. Hence, in the weak-field limit, the branching ratio $\mathrm{OD} / \mathrm{OH}$ in Eq. (1) obtained with any form of UV-pulse shaping will always be larger than 1.9.

Some time ago, it was suggested that laser light can create and guide nuclear wave packets via properly timed pump and dump pulses between various potential energy surfaces in molecules [28]. That is, one takes advantage of the different forces which operate in different electronic states. We apply this strategy and note that in the strong-field limit, amplitude can be transferred from the ground state to the excited state surface and back to the ground state surface, and so on.

In practice there are various constraints which must be taken into consideration when we leave the weak-field limit. An estimate of the threshold intensity for ionization [29] gives $I \sim 100 \mathrm{TW} / \mathrm{cm}^{2}$ and in order to avoid significant ionization, we consider in the following only intensities below this limit. At very high intensities the two-surface model of HOD is not reliable and, in general, we want intensities as low as possible in order to avoid the opening of unwanted reaction channels including ionization channels.

One conceivable mechanism, eventually leading to preferred cleavage of the O-D bond, would be to manipulate the wave packet as it evolves on the potential energy surface of
TABLE I. Branching ratios for various optimized pulses.

\begin{tabular}{lc}
\hline$I /\left(\mathrm{TW} / \mathrm{cm}^{2}\right)$ & $(\mathrm{H}+\mathrm{OD}) /(\mathrm{D}+\mathrm{OH})$ \\
8 & 1.9 \\
18 & 1.2 \\
56 & 0.5 \\
\hline \hline
\end{tabular}

the excited electronic state. Thus, a dump pulse could remove parts of the wave packet such that what is left of the original wave packet, evolves as desired.

To that end, the branching ratio has been analyzed for Gaussian (transform-limited) pulses as a function of temporal duration at various field strengths [19]. It was observed that, for short pulses, high field strengths imply a lowering in the branching ratio compared to the weak-field limit. The mechanism of this bond-selective dumping could be described as suggested above [19]. However, we observe that at the highest field strengths $\left(56 \mathrm{TW} / \mathrm{cm}^{2}\right)$, the smallest branching ratio $\mathrm{OD} / \mathrm{OH}$ is still close to 1.9 , as obtained in the $\mathrm{cw}$ limit for weak fields. We note in passing that if the intensity of the transform-limit pulse is further increased by a factor of 10-20, the branching ratio is reduced below 1.9, see also Ref. [18], but at these "extreme" intensities the twosurface model is not expected to be reliable. Thus, the application of nontrivial shaped pulses seems to be essential in order to avoid extreme laser intensities.

All results reported in the following are based on the description of HOD given in Ref. [14]. We note that the magnitude of the transition-dipole moment decreases as a function of the bond lengths making it increasingly more difficult to transfer wave packet amplitude at larger distances (the transition-dipole moment was often assumed to be constant in previous model studies). The wave packet propagation is carried out using two $512 \times 512$ grids with a grid spacing of 0.05 a.u. The action of the kinetic energy operator is evaluated by the FFT method, and the time propagation is done by the split-operator method with a time step of 1 a.u. [30].

The optimized laser pulse shape is calculated via optimal control theory [30-32] with the objective of maximizing population in the $\mathrm{D}+\mathrm{OH}$ channel under the constraint of a constant energy of the laser field. Since, ideally, we want both a high selectivity and a high yield, the target state is one where all population ends up in the $\mathrm{D}+\mathrm{OH}$ channel of the excited electronic state. The target time is set to 2000 a.u. ( $\sim 50 \mathrm{fs})$. The initial pulse is taken to be a Gaussian (5 fs FWHM $)$ with center frequency $\omega=0.2738$ a.u. $\quad(\lambda$ $=166.5 \mathrm{~nm})$. The optimized laser pulse is obtained by an iterative procedure [32], all results in the following were converged after 15 iterations.

The molecule is aligned such that the transition dipolemoment vector is parallel to the electric field vector of the (plane polarized) laser field. The branching ratios obtained at various maximum intensities (cycle-averaged peak intensities $I=\epsilon_{0} c E_{0}^{2} / 2$ ) are shown in Table I. It is seen that it is possible to obtain preferential breaking of the O-D bond, even when HOD initially is in its vibrational ground state. The threshold for breaking the lower limit of the branching ratio of the weak-field limit is $10-12 \mathrm{TW} / \mathrm{cm}^{2}$. 

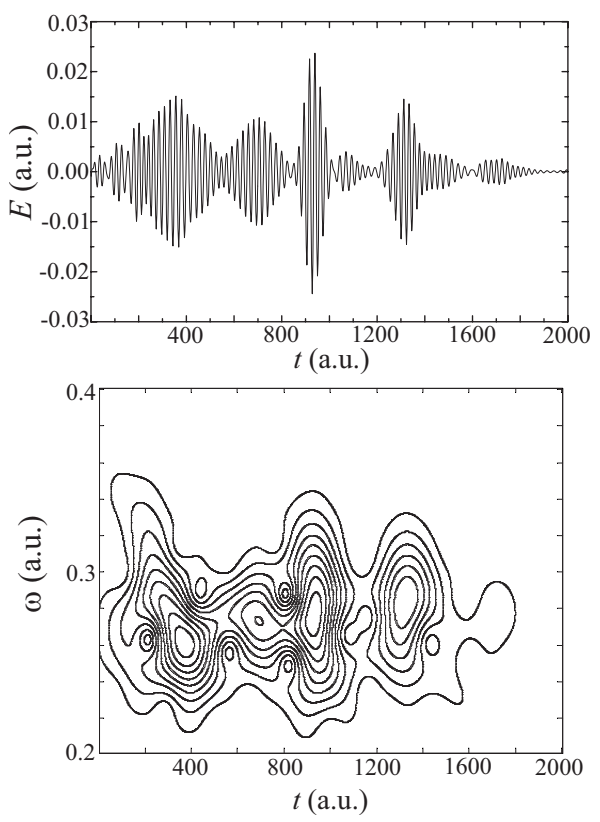

FIG. 2. The upper panel shows the optimized pulse (cycleaveraged peak intensity $18 \mathrm{TW} / \mathrm{cm}^{2}$ ). The lower panel shows the Husimi transform of the pulse.

We now analyze, in more detail, the case where $\mathrm{OD} / \mathrm{OH}=1.2$. The upper panel in Fig. 2 shows the optimized pulse. The cycle-averaged peak intensity (around 950 a.u.) is $18 \mathrm{TW} / \mathrm{cm}^{2}$, however, the average intensity of the entire pulse is somewhat lower. The lower panel shows the Husimi transform of the pulse, i.e., the time-frequency behavior of the pulse, obtained as the overlap between the pulse and a continuous family of complex Gaussians $\left(\alpha=10^{-4}\right.$ a.u.) [30]. The bandwidth covers, essentially, the entire first absorption band. A positive chirp is observed in the first part of the pulse. Positively chirped pulses have previously been found to efficiently transfer amplitude to excited states [33].

The upper panel in Fig. 3 shows the populations in the ground and excited state for the pulse in Fig. 2. We observe that more than $90 \%$ of the population has been transferred to the excited state. The lower panel shows the time-resolved "branching ratio," i.e., the ratio between the populations in each channel (defined according to the dividing line where the two bond lengths are equal). This ratio becomes the branching ratio within about 5-6 fs (250 a.u.) after the pulse has decayed. Comparing with Fig. 2, we observe that the pulse features after about 1000 a.u. basically are insignificant. Note that if only the first part of pulse up to 825 a.u. is used, a branching ratio of 1.83 is obtained.

We now turn to the interpretation of the results. Figure 4 displays the wave function (absolute square) in the electronic ground state for the pulse in Fig. 2. Clearly, nonstationary vibrational states are created involving combinations of $\mathrm{O}-\mathrm{H}$ and O-D stretches, with a positive momentum in the O-D stretch. If the wave packet on the ground state at 950 a.u. is excited with a $\delta$ pulse, a branching ratio of 0.75 is obtained. Thus, the momentum corresponding to stretching of the O-D bond is transferred to the excited state. Roughly speaking, the first part of the pulse (up to 825 a.u.) transfers about $50 \%$

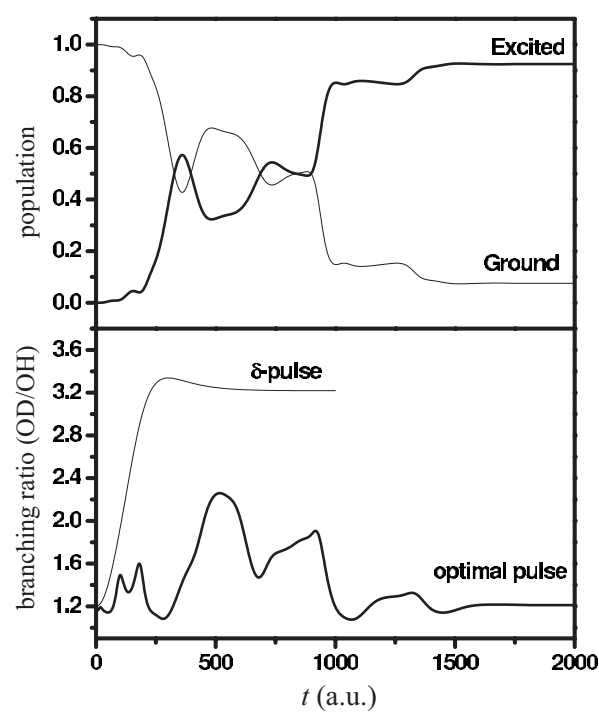

FIG. 3. The upper panel shows the population in the ground and excited state for the pulse in Fig. 2. The lower panel shows the time-resolved "branching ratio," see text. The result corresponding to excitation by an infinitely short $\delta$ pulse is also shown.

of the population and with approximately the remaining 50\% population transferred by the short pulse around 950 a.u., the average branching ratio can be estimated to $(1.83+0.75) / 2$ $=1.29$, in good agreement with the 1.2 reported above.

The mechanism, in its simplest form, involves a "pumpdump-pump" sequence. As shown above the key point in the mechanism is the creation of the nonstationary vibrational state. This is similar to the two-pulse control scheme suggested some time ago [14], where an (intense) IR pulse forces vibrational motion in the molecule and, at the optimal delay time, a UV pulse transfers this wave packet to the excited state. One advantage of this approach is that excitation to the excited state takes place, when the initial state is optimal with respect to position and/or momentum.

For HOD, it is a very challenging task to beat the selectivity obtained within traditional photochemistry with weak

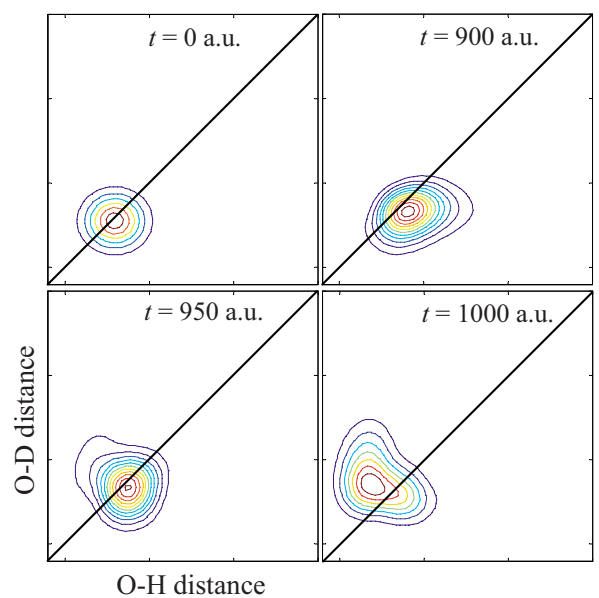

FIG. 4. (Color online) Plots of the vibrational probability density in the electronic ground state during the action of the pulse in Fig. 2. 
cw-light sources. However, the present work suggests that through careful optimization of pulse shapes, it is possible to obtain branching ratios which are not accessible in the weakfield cw limit. The optimized pulses can, in addition, produce products at a yield close to $100 \%$.

Pulse shaping techniques in the relevant ultraviolet region are under development [34]. This technique is essential in order to avoid extreme laser intensities but, as shown in this work, high intensities might still be required. An interesting general question is to what extent laser controlled selective bond breakage is possible without simultaneous ionization of molecules.

The Carlsberg foundation and the Danish National Research Foundation's Center for Molecular Movies (CMM) are acknowledged for financial support.
[1] N. E. Henriksen and F. Y. Hansen, Theories of Molecular Reaction Dynamics (Oxford University Press, Oxford, 2008).

[2] S. A. Rice and M. Zhao, Optical Control of Molecular Dynamics (Wiley, New York, 2000).

[3] H. Rabitz, R. de Vivie-Riedle, M. Motzkus, and K. Kompa, Science 288, 824 (2000).

[4] A. Assion, T. Baumert, M. Bergt, T. Brixner, B. Kiefer, V. Seyfried, M. Strehle, and G. Gerber, Science 282, 919 (1998).

[5] T. C. Weinacht, J. Ahn, and P. H. Bucksbaum, Nature (London) 397, 233 (1999).

[6] T. Brixner, N. H. Damrauer, P. Niklaus, and G. Gerber, Nature (London) 414, 57 (2001).

[7] R. J. Levis, G. M. Mekir, and H. Rabitz, Science 292, 709 (2001).

[8] G. Vogt, G. Krampert, P. Niklaus, P. Nuernberger, and G. Gerber, Phys. Rev. Lett. 94, 068305 (2005).

[9] C. Daniel, J. Full, L. Gonzalez, C. Lupulescu, J. Manz, A. Merli, S. Vajda, and L. Wöste, Science 299, 536 (2003).

[10] J. Zhang, D. G. Imre, and J. H. Frederick, J. Phys. Chem. 93, 1840 (1989).

[11] I. Bar, Y. Cohen, D. David, S. Rosenwaks, and J. J. Valentini, J. Chem. Phys. 93, 2146 (1990).

[12] R. L. Vander Wal, J. L. Scott, and F. F. Crim, J. Chem. Phys. 92, 803 (1990).

[13] R. L. Vander Wal, J. L. Scott, F. F. Crim, K. Weide, and R. Schinke, J. Chem. Phys. 94, 3548 (1991).

[14] B. Amstrup and N. E. Henriksen, J. Chem. Phys. 97, 8285 (1992).

[15] N. E. Henriksen and B. Amstrup, Chem. Phys. Lett. 213, 65 (1993).

[16] K. Mishima and K. Yamashita, J. Chem. Phys. 110, 7756 (1999).
[17] N. Elghobashi, P. Krause, J. Manz, and M. Oppel, Phys. Chem. Chem. Phys. 5, 4806 (2003).

[18] M. Sarma, S. Adhikari, and M. K. Mishra, J. Chem. Phys. 127, 024305 (2007).

[19] K. B. Møller, H. C. Westtoft, and N. E. Henriksen, Chem. Phys. Lett. 419, 65 (2006).

[20] V. Engel and R. Schinke, J. Chem. Phys. 88, 6831 (1988).

[21] R. Schinke, Photodissociation Dynamics (Cambridge University Press, Cambridge, 1993).

[22] N. E. Henriksen, K. B. Møller, and V. Engel, J. Chem. Phys. 122, 204320 (2005)

[23] N. Shafer, S. Satyapal, and R. Bersohn, J. Chem. Phys. 90, 6807 (1989).

[24] A. K. Tiwari, K. B. Møller, and N. E. Henriksen, Chem. Phys. Lett. 450, 6 (2007).

[25] P. Brumer and M. Shapiro, Chem. Phys. 139, 221 (1989).

[26] M. Shapiro and P. Brumer, J. Phys. Chem. A 105, 2897 (2001).

[27] N. E. Henriksen, Adv. Chem. Phys. 91, 433 (1995).

[28] D. J. Tannor and S. A. Rice, J. Chem. Phys. 83, 5013 (1985).

[29] P. Dietrich and P. B. Corkum, J. Chem. Phys. 97, 3187 (1992).

[30] D. J. Tannor, Introduction to Quantum Mechanics (University Science Books, New York, 2007).

[31] R. Kosloff, S. A. Rice, P. Gaspard, S. Tersigni, and D. J. Tannor, Chem. Phys. 139, 201 (1989).

[32] J. Somlói, V. A. Kazakov, and D. J. Tannor, Chem. Phys. 172, 85 (1993).

[33] J. Cao, C. Bardeen, and K. R. Wilson, J. Chem. Phys. 113, 1898 (2000).

[34] P. Nuernberger, G. Vogt, R. Selle, S. Fechner, T. Brixner, and G. Gerber, Appl. Phys. B 88, 519 (2007). 